\title{
La loi interdisant la dissimulation du visage dans l'espace public et les limites du contrôle pratiqué par le Conseil constitutionnel ${ }^{*}$
}

\author{
Aurore Gaillet \\ Maître de conférences \\ à l'Université de Strasbourg
}

Si le nouveau mécanisme de question prioritaire de constitutionnalité participe assurément à la définition d'un nouveau paysage juridique et juridictionnel, la saisine a priori du Conseil constitutionnel n'est pas pour autant tombée en désuétude. Son intérêt persistant pour les autorités de saisine est souligné par la décision du 7 octobre 2010, laquelle juge conforme à la Constitution, à une réserve d'interprétation près, la loi interdisant la dissimulation du visage dans l'espace public ${ }^{1}$.

Les controverses liées à la pratique du port du voile intégral «sur le territoire de la République française $»^{2}$ ont, dans un premier temps, été à l'origine de la création d'une mission d'information parlementaire créée à ce sujet le 23 juin 2009. Tout en relevant que «la loi est le seul vecteur normatif possible pour interdire le voile intégral dans l'espace public», le rapport précise toutefois qu'il «n'existe pas d'unanimité pour l'adoption d'une loi d'interdiction générale et absolue »3. Partant, les députés ont choisi d'exprimer une position

* La rédaction de cet article a été achevée en décembre 2011.

1. Décision $\mathrm{n}^{\circ}$ 2010-613 DC du 7 octobre 2010 - Loi interdisant la dissimulation du visage dans l'espace public; Loi $\mathrm{n}^{\circ}$ 2010-1192, 11 octobre 2010, J0 12 octobre 2010.

2. Les termes du président de la République, déclarant devant le Congrès réuni à Versailles, le 22 juin 2009 que «la burqa n'est pas la bienvenue sur le territoire de la République française» ont engagé le débat. Sur les différentes étapes ayant mené à la promulgation de la loi : A. Levade, «Épilogue d'un débat juridique: l'interdiction de la dissimulation du visage dans l'espace public validée!», JCP G, n 43,25 octobre 2010, p. 1043.

3. "Voile intégral: le refus de la République» présenté par le président A. Gérin et le rapporteur E. Raoult, Rapport d'information au nom de la mission d'information sur la pratique du port du voile intégral sur le territoire national, $\mathrm{n}^{\circ}$ 2262, 26 janvier 2010, p. 187-188. Voir aussi: A. Levade, «"Le refus de la République”, prologue d’un débat national? - À propos du rapport de la mission 
politique, en adoptant à l'unanimité des 434 suffrages exprimés (435 votants, une abstention) une résolution «sur l'attachement au respect des valeurs républicaines face au développement de pratiques radicales qui y portent atteinte $»^{4}$. Rappelons qu'une telle résolution est fondée sur l'article 34-1 de la Constitution, introduit par la loi constitutionnelle du 23 juillet 2008 à l'effet de permettre aux assemblées d'exprimer une opinion générale. S'ensuit, en principe, une stricte distinction entre la loi, normative, et les résolutions qui, comme en l'espèce, sont privées de toute force obligatoire. Dissocié du processus législatif, le vote de la résolution aurait pu concourir à la lutte contre «l'inflation législative [...] motivée par la course à la médiatisation et nourrie par l'instrumentalisation des peurs $»^{5}$. Le lien entre le vote de la résolution le 11 mai 2010 et l'adoption d'un projet de loi «interdisant la dissimulation du visage dans l'espace public» le 19 mai n'en est pas moins direct.

Après avoir élargi son objet, afin d'éviter le grief de discrimination ou de stigmatisation de la communauté musulmane, la loi dispose notamment que «nul ne peut, dans l'espace public, porter une tenue destinée à dissimuler son visage» (article $1^{\mathrm{er}}$ ). Ni l'absence d'accord politique, manifesté par le refus d'une majorité de députés et de sénateurs de gauche de prendre part au vote, ni l'incertitude juridique entourant une prohibition générale et absolue n'ont empêché son adoption par l'Assemblée nationale le 13 juillet, puis par le Sénat le 14 septembre. Parachevant ce parcours législatif atypique, la décision rendue par le Conseil constitutionnel, aussitôt saisi le 14 septembre, permetelle de mettre fin au débat? Tel est en tout cas l'un des objectifs visés par les auteurs de la saisine.

La décision laisse néanmoins le lecteur perplexe. Rendue au terme d'une saisine a priori et abstraite, elle apparaît juridiquement décevante.

d'information sur la pratique du port du voile intégral sur le territoire national », JCP G, $\mathrm{n}^{\circ} 6,8$ février 2010, p. $142 \mathrm{~s}$.

4. Assemblée nationale, xIII législature, $\mathrm{TA}^{\circ}{ }^{\mathrm{e}}$ 459, 11 mai 2010.

5. J.-P. Feldman, «Burqa: une loi dangereuse et inutile», Recueil Dalloz, 2010, p. 387. 


\section{Une saisine a priori et abstraite : de l'usage d'un dispositif ancien dans un contexte juridique nouveau}

Eu égard aux doutes formulés quant à la constitutionnalité de la loi, la saisine préventive du Conseil constitutionnel fut rapidement envisagée par l'auteur de la proposition de loi lui-même. Pour Jean-François Copé, alors président du groupe majoritaire à l'Assemblée nationale, il s'agissait de «lever » rapidement ces «doutes», "de sorte que [1']application [de la loi] ne puisse pas être contestée $»^{6}$. Quoique soutenue au-delà des seuls rangs de la majorité$^{7}$, cette procédure revêt de toute évidence un caractère politique. La saisine a priori se justifie certainement en partie par l'existence même du nouveau mécanisme de question prioritaire de constitutionnalité. Reste que la décision coupe court sur ce point à la nouvelle logique d'appropriation de la Constitution par les citoyens.

\section{La saisine du Conseil constitutionnel : une parade politique?}

La décision du 7 octobre 2010 est issue d'une saisine conjointe des présidents des deux assemblées, dans des conditions qui ramènent à la rédaction initiale de l'article 61, laquelle n'ouvrait la porte du Conseil constitutionnel qu'à des autorités politiques. Préventive et abstraite, la saisine tranche avec l'évolution observée depuis l'entrée en vigueur de la révision du 23 juillet 2008.

\section{La première saisine conjointe du Conseil constitutionnel par les présidents des deux assemblées}

«Monsieur le Président, En application du deuxième alinéa de l'article 61 de la Constitution, j'ai l'honneur de soumettre au Conseil constitutionnel la loi interdisant la dissimulation du visage dans l'espace public, en vue de l'examen de sa conformité à la Constitution. Je vous prie d'agréer, Monsieur le Président, l'expression de ma haute considération». Rédigée en des termes identiques par le président de l'Assemblée nationale, Bernard Accoyer, et par le président du Sénat, Gérard Larcher, cette saisine fait de la loi sur la

6. JOAN CR, 8 juillet 2010, p. 5415.

7. Voir ainsi Robert Badinter: «[...] quel que soit le texte voté, il faut que le président du Sénat, comme celui de l'Assemblée nationale, le défère au Conseil constitutionnel» (VO Sénat CR, 15 septembre 2010, $n^{\circ} 82$ S, p. 6760). 
dissimulation du visage dans l'espace public la première loi déférée concomitamment par les présidents des deux assemblées en application de l'article 61 al. 2 de la Constitution.

Le rappel des rares saisines formées respectivement par le président du Sénat et par le président de l'Assemblée nationale met en lumière l'originalité de cette procédure.

La limitation originelle des modalités de saisine du Conseil constitutionnel s'expliquait, on le sait, par sa mission $\mathrm{d}^{\prime}$ « organe régulateur des pouvoirs publics ${ }^{8}$, relevant davantage d'une institution modeste du système politique que d'une cour constitutionnelle. Dans ce contexte, seul le président du Sénat fut en mesure de s'ériger contre "l'arbitraire de la majorité»", un arbitraire dont la possibilité était renforcée par le phénomène majoritaire de la Ve République. Tel est l'esprit qui anima les saisines ayant conduit aux décisions du 6 novembre 1962, du 16 juillet 1971 et du 27 décembre $1973^{10}$. Le droit de saisir le Conseil constitutionnel ayant été reconnu à la minorité, ce rôle spécifique, joué par le président du Sénat, s'effaça. Après la révision constitutionnelle du 29 octobre 1974, trois saisines du président du Sénat peuvent encore être mentionnées. Elles accompagnent cependant les saisines de soixante députés ${ }^{11}$, voire, pour les décisions du 2 décembre 1982 et du 9 mai 1991, les saisines de soixante députés et de soixante sénateurs ${ }^{12}$. On peut y déceler la volonté de souligner les doutes émis quant à la constitutionnalité des lois déférées - au reste censurées au moins partiellement par le Conseil constitutionnel. Quant aux trois saisines formées par le président de l'Assemblée nationale, elles répondent également à des difficultés juridiques et politiques souvent importantes ${ }^{13}$. Les querelles relatives à l'examen et au

8. Décision n 62-20 DC du 06 novembre 1962 - Loi relative à l'élection du Président de la République au suffrage universel direct, adoptée par le référendum du 28 octobre 1962 (considérant 2).

9. H. Kelsen, La démocratie, sa nature, sa valeur, (1929), trad. fr. C. Eisenmann, Paris, Economica, 1988, p. 73.

10. Décision précitée $n^{\circ}$ 62-20 DC du o6 novembre 1962; Décision n 71-44 DC du 16 juillet 1971 - Loi complétant les dispositions des articles 5 et 7 de la loi du $1^{\text {er }}$ juillet 1901 relative au contrat d'association; Décision n 73-51 DC du 27 décembre 1973 - Loi de finances pour 1974.

11. Décision no 83-168 DC du 20 janvier 1984 - Loi portant dispositions statutaires relatives à la fonction publique territoriale.

12. Décision $n^{\circ}$ 82-147 DC du 02 décembre 1982 - Loi portant adaptation de la loi $n^{\circ}$ 82-213 du 02 mars 1982 relative aux droits et libertés des communes, des départements et des régions à la Guadeloupe, à la Guyane, à la Martinique et à la Réunion; Décision n 91-290 DC du o9 mai 1991 - Loi portant statut de la collectivité territoriale de Corse.

13. Sur ces questions: P. Jan, La saisine du Conseil constitutionnel, Paris, L.G.D.J., «Bibliothèque constitutionnelle et de science politique», t. 93, 1999. 
vote du projet de loi de finances pour 1980 ont en particulier conduit à deux recours, portant sur un même texte, formés par le président de l'Assemblée nationale et par des députés de l'opposition ${ }^{14}$. Contrairement à ces derniers, le président Chaban-Delmas ne requérait pas expressément une déclaration de non-conformité de la loi. Il importait à ses yeux de conduire le Conseil constitutionnel à interpréter une disposition de valeur constitutionnelle et à se prononcer sur la régularité de la procédure budgétaire controversée. «En réalité, il s'agit davantage d'une demande de consultation ${ }^{15}$, la compétence consultative du Conseil constitutionnel étant par ailleurs fortement limitée ${ }^{16}$. Lorsque le président Philippe Séguin décide de soumettre les deux lois dites «bioéthique» de 1994 à l'examen du Conseil constitutionnel, il se place certes dans une logique quelque peu différente. «Eu égard à la portée déterminante de ces textes pour la mise en œuvre de libertés et de droits fondamentaux qui procèdent de principes et de règles à valeur constitutionnelle, [il invite le Conseil à se prononcer] afin que leur conformité à la Constitution ne puisse être affectée d'aucune incertitude $»^{17}$. La recherche d'apaisement qui l'anime n'est cependant pas partagée par les soixante députés qui, se fondant sur de très nombreux griefs, mettent par ailleurs en doute la constitutionnalité de la loi.

La saisine ayant donné lieu à la décision du 7 octobre 2010 a ceci de particulier qu'elle se situe hors de toute stratégie d'opposition. Sollicitée par un membre du parti politique à l'origine de la loi, elle ne tend pas à modérer les excès de la majorité, mais bien davantage à apporter une caution juridique à une loi contestée.

14. Décision $n^{\circ} 79-110$ DC du 24 décembre 1979 - Loi de finances pour 1980. La décision $n^{\circ}$ 76-74 DC du 28 décembre 1976 - Loi de finances rectificative pour 1976 et notamment ses articles 6, 10, $11,12,13,14,15,16,17,18$ et 22 est issue d'un recours, plus classique, formé par le seul président de l'Assemblée nationale.

15. L. Favoreu, L. Philip, Les grandes décisions du Conseil constitutionnel, Paris, Dalloz, $15^{\mathrm{e}}$ éd., 2009, $n^{\circ} 26$. Était notamment en jeu l'interprétation de l'article 40 de l'ordonnance portant loi organique du 2 janvier 1959 relative aux lois de finances.

16. Décision du 14 septembre 1961 - Demande d'avis présentée par le président de l'Assemblée nationale (Recevabilité de la motion de censure).

17. Lettre de transmission du président de l'Assemblée nationale, Décision n 94-343/344 DC du 27 juillet 1994 - Loi relative au respect du corps humain et loi relative au don et à l'utilisation des éléments et produits du corps humain, à l'assistance médicale à la procréation et au diagnostic prénatal. 


\section{Une saisine préventive et abstraite}

Sans doute peut-on saluer la saisine du Conseil constitutionnel, annoncée par le président du groupe UMP à l'Assemblée, alors même que l'opposition avait précisé qu'elle ne ferait pas «obstacle à la loi» en utilisant le droit de saisine, classiquement actionné par les parlementaires de la minorité. Une volonté d'éviter le contrôle de constitutionnalité, telle qu'affichée lors du vote de la loi n 2004-228 du 15 mars 2004 «encadrant, en application du principe de laïcité, le port de signes ou de tenues manifestant une appartenance religieuse dans les écoles, collèges et lycées publics ${ }^{18}$, semble bien être vouée à disparaître des calculs politiques. L'un des arguments en faveur de l'introduction d'un mécanisme d'exception d'inconstitutionnalité dans la Constitution française tenait précisément au caractère facultatif du contrôle des lois ordinaires $^{19}$.

Sur ce point, le progrès pour l'État de droit est indéniable: la nécessité de tenir compte du contrôle de constitutionnalité est définitivement intégrée par les acteurs du processus législatif. Un refus de saisine procédant d'un accord au moins tacite des parlementaires n'est plus insurmontable. L'existence même du nouveau dispositif, en vigueur depuis le $1^{\text {er }}$ mars 2010, permettant à tout justiciable de contester la conformité d'une disposition législative aux droits et libertés constitutionnels a incité les auteurs de la loi à transmettre ce dossier au Conseil constitutionnel, de manière préventive. Il était en effet fort probable que la première personne se voyant sanctionnée pour avoir porté une tenue «destinée à dissimuler son visage» eût, lors d'un potentiel procès, argué de l'inconstitutionnalité de la loi. Le consensus politique ne saurait s'apparenter à un critère de conformité au droit. L'un des arguments classiques en faveur de l'exception d'inconstitutionnalité se trouve alors vérifié.

Les incertitudes juridiques soulevées lors du débat parlementaire ont ainsi justifié le contrôle de la loi avant sa promulgation par le président de la République. Reste qu'un tel contrôle préventif, effectué immédiatement après le débat politique, ne manque pas de marquer la décision d'un sceau politique. La logique même d'une saisine a priori a, de surcroît, contraint le Conseil constitutionnel à statuer avant que les effets de la loi contrôlée n'aient pu être observés et évalués.

18. Sur ce point: R. Libchaber, «À la croisée des interprétations: le voile et la loi », RTDciv., 2004, p. 162.

19. G. Vedel, «Réflexions sur les singularités de la procédure devant le Conseil constitutionnel», in Mélanges Roger Perrot, Paris, Dalloz, 1995, p. 537s. 
Or, quel que soit le caractère novateur de la nouvelle procédure de contrôle a posteriori, elle n'ouvre pas, en l'espèce, de véritable perspective pour une nouvelle contestation de la loi sur le terrain de sa constitutionnalité.

\section{L'absence de grief juridique contre la loi : vers un brevet de constitutionnalité excluant une saisine $a$ posteriori}

La décision rendue par le Conseil constitutionnel, amené à se prononcer sur une loi contre laquelle aucun grief précis n'est formulé, court-circuite toute éventuelle question prioritaire de constitutionnalité en la matière.

\section{Une curieuse saisine «blanche»}

«Considérant que le président de l'Assemblée nationale et le président du Sénat défèrent au Conseil constitutionnel la loi interdisant la dissimulation du visage dans l'espace public; qu'ils n'invoquent à l'encontre de ce texte aucun grief particulier» (considérant 1). La possibilité de former un recours sans développer aucune argumentation juridique ne manque pas de soulever des interrogations quant à la qualité juridictionnelle de l'instance saisie. Cette obligation est en effet réputée contribuer à la bonne administration de la justice. Devant le juge administratif par exemple, si l'exigence de motivation des recours relève d'un régime souvent qualifié de libéral ${ }^{20}$, la requête n'est recevable que si elle contient «l'exposé des faits et moyens, ainsi que l'énoncé des conclusions soumises au juge» (article R 411-1 CJA).

Il est vrai que cette obligation n'est pas prévue s'agissant de la saisine formée sur la base de l'article 61 al. 2 de la Constitution. La logique d'une procédure à finalité purement objective, dans laquelle aucun droit subjectif n'est en cause, autorise cet usage des saisines non motivées, dont l'objectif est d'obtenir un brevet de constitutionnalité au profit du texte déféré et non de le contester, de porter devant le Conseil un véritable contentieux. Dans le contexte créé par l'article 61-1 de la Constitution, cet usage prend toutefois une signification nouvelle. L'exigence de la motivation des questions prioritaires de constitutionnalité souligne, a contrario, le caractère systématique de cet impératif en matière juridictionnelle ${ }^{21}$.

20. R. Chapus, Droit du contentieux administratif, Paris, Montchrestien, $13^{\mathrm{e}}$ éd., 2008, p. $500 \mathrm{~s}$. 21. Article 23-1 de la loi organique $n^{\circ} 2009-1523$ du 10 décembre 2009 relative à l'application de l'article 61-1 de la Constitution, complétant l'ordonnance $n^{\circ}$ 58-1067 du 7 novembre 1958 portant loi organique sur le Conseil constitutionnel: «Devant les juridictions relevant du Conseil d’État ou de la Cour de cassation, le moyen tiré de ce qu'une disposition législative porte atteinte aux droits 
La logique suivie par la saisine du 14 septembre 2010 n'est en effet pas à confondre avec d'autres «saisines blanches » par lesquelles une autorité politique, le plus souvent le président de la République, «demand[e], au Conseil constitutionnel [au titre de l'article 54 de la Constitution] si l'autorisation de ratifier [un] traité doit être précédée d'une révision de la Constitution ${ }^{22}$. L'absence de motivation de la saisine s'inscrit alors dans le cadre particulier de la ratification des traités, notamment ceux destinés à renforcer la construction européenne. De même, les auteurs de la saisine ici étudiée n'aspirent pas, à l'instar des parlementaires d'opposition à l'origine de la décision sur la loi pénitentiaire ${ }^{23}$, à présenter une contestation systématique de la loi déférée. Le défaut de motivation révèle au contraire le soutien apporté à la loi. Ce choix tranche précisément avec l'abondance des arguments juridiques mis en avant lors du débat législatif. Renonçant à articuler un grief quelconque à l'encontre de la loi, une telle saisine «blanche» n'a rien d'un «procès fait à la loi », lequel aurait $\mathrm{pu}$, de manière bien plus explicite, initier un véritable débat devant le Conseil constitutionnel. Si le débat n'est pas «lancé» par la lettre de saisine, il aurait toutefois pu avoir lieu devant le Conseil constitutionnel, lequel s'estime traditionnellement saisi in rem, c'est-à-dire de l'intégralité du texte, la constitutionnalité s'appréciant en l'ensemble de ses éléments, y compris ceux non invoqués par les auteurs de la saisine.

Une saisine si large aurait ainsi pu justifier qu'il soit procédé à un examen méticuleux de l'ensemble de la loi au regard de l'ensemble de la Constitution. Loin de là. La brièveté de la décision, ne comportant qu'une motivation extrêmement ténue déçoit. À l'issue de six considérants peu développés, la loi, à une réserve près, est «déclarée conforme à la Constitution».

et libertés garantis par la Constitution est, à peine d'irrecevabilité, présenté dans un écrit distinct et motivé. [...]»; Article 23-5: «Le moyen tiré de ce qu'une disposition législative porte atteinte aux droits et libertés garantis par la Constitution peut être soulevé, y compris pour la première fois en cassation, à l'occasion d'une instance devant le Conseil d'État ou la Cour de cassation. Le moyen est présenté, à peine d'irrecevabilité, dans un mémoire distinct et motivé. [...]»; Article 23-7: «La décision motivée du Conseil d'État ou de la Cour de cassation de saisir le Conseil constitutionnel lui est transmise avec les mémoires ou les conclusions des parties.».

22. Voir par exemple le texte de la saisine à l'origine de la décision $n^{\circ}$ 2007-560 DC du 20 décembre 2007 - Traité de Lisbonne modifiant le traité sur l'Union européenne et le traité instituant la Communauté européenne.

23. Décision n 2009-593 DC du 19 novembre 2009 - Loi pénitentiaire: «Conformément au second alinéa de l'article 61 de la Constitution, nous avons l'honneur de déférer au Conseil constitutionnel l'ensemble de la loi pénitentiaire [...]». Voir à ce sujet: "Décision relative à la loi pénitentiaire: désaveu de la saisine blanche (décision $n^{\circ}$ 2009-593 DC du 19 novembre 2009) »:

http://combatsdroitshomme.blog.lemonde.fr/2009/11/24/decision-relative-a-la-loi-penitentiairedesaveu-de-la-saisine-blanche-decision-n²009-593-dc-du-19-novembre-2009. 
À ce stade de l'analyse, il importe de comprendre dans quelle mesure, cette décision court-circuite, pour un temps du moins, la possibilité d'une saisine ultérieure dans le cadre du mécanisme de question prioritaire de constitutionnalité.

\section{La question prioritaire de constitutionnalité court-circuitée}

On a pu se demander si la loi entrée en vigueur le 11 octobre 2010 pourrait faire l'objet d'un contrôle ultérieur, à l'occasion d'une question prioritaire de constitutionnalité posée dans le cadre d'un débat contradictoire. En effet, le critère posé par l'article 23-2 de la loi organique du 10 décembre 2009 relative à l'application de l'article 61-1, selon lequel la question prioritaire de constitutionnalité ne peut être transmise que si «elle n'a pas déjà été déclarée conforme à la Constitution dans les motifs et le dispositif d'une décision du Conseil constitutionnel, sauf changement des circonstances $»^{24}$ semble s'opposer à une telle éventualité. Ainsi que l'a souligné le Conseil dans sa décision du 3 décembre 2009, ce critère rappelle l'autorité de ses décisions, énoncée par le dernier alinéa de l'article 62 de la Constitution ${ }^{25}$.

La règle selon laquelle il ne peut y avoir de question prioritaire de constitutionnalité si la disposition contestée a déjà été déclarée conforme "dans les motifs et le dispositif " d'une décision a déjà été appliquée à plusieurs reprises $^{26}$. Dans un arrêt rendu le 19 mai 2010, le Conseil d'État considère par exemple que la déclaration de conformité d'une disposition législative ne fait pas obstacle au renvoi d'un article dont le Conseil constitutionnel «n'a pas expressément examiné la constitutionnalité [...] dans les motifs de sa décision ${ }^{27}$. Pour le Conseil constitutionnel, le mieux placé pour apprécier la

24. Loi organique $n^{\circ}$ 2009-1523 du 10 décembre 2009 (précitée).

25. Décision $\mathrm{n}^{\circ}$ 2009-595 DC du 3 décembre 2009 - Loi organique relative à l'application de l'article 61-1 de la Constitution: "Considérant, en premier lieu, que les trois conditions qui déterminent la transmission de la question prioritaire de constitutionnalité ne méconnaissent pas l'article 61-1 de la Constitution; que la condition prévue par le $2^{\circ}$ de l'article $23-2$ est conforme au dernier alinéa de l'article 62 de la Constitution qui dispose: "Les décisions du Conseil constitutionnel ne sont susceptibles d'aucun recours. Elles s'imposent aux pouvoirs publics et à toutes les autorités administratives et juridictionnelles"; qu'en réservant le cas du "changement des circonstances”, elle conduit à ce qu'une disposition législative déclarée conforme à la Constitution dans les motifs et le dispositif d'une décision du Conseil constitutionnel soit de nouveau soumise à son examen lorsqu'un tel réexamen est justifié par les changements intervenus, depuis la précédente décision, dans les normes de constitutionnalité applicables ou dans les circonstances, de droit ou de fait, qui affectent la portée de la disposition législative critiquée» (considérant 13).

26. P. Bon, «Premières questions, premières précisions », RFDA, 2010, p. 679.

27. CE, 19 mai 2010, Section française de l'Observatoire international des prisons, req. $\mathrm{n}^{\circ} 323930$. Nous soulignons. 
portée de ses propres décisions, la condition de recevabilité n'est pas satisfaite dans de telles circonstances. Il renvoie à cet effet au «dispositif» de sa décision, dont l'un des articles «a déclaré [la disposition litigieuse] conforme à la Constitution ${ }^{28}$. Pour la loi qui nous occupe ici, l'invocation d'un motif d'inconstitutionnalité non couvert par l'autorité de la chose jugée semble difficile tant la rédaction de la décision du Conseil constitutionnel est large. Dès lors que «[s]ous la réserve énoncée au considérant 5, la loi interdisant la dissimulation du visage dans l'espace public est conforme à la Constitution », il serait vraisemblablement considéré que la décision du 7 octobre 2010 vise, dans son dispositif, l'ensemble de la loi et embrasse l'examen de tous ses articles.

De même, l'exception des circonstances nouvelles ne saurait sans doute jouer avant longtemps. Dès sa décision précitée du 3 décembre 2009, le Conseil constitutionnel a précisé que cette dernière notion vise les hypothèses dans lesquelles un réexamen de la disposition déclarée conforme s'avère «justifié par les changements intervenus, depuis la précédente décision, dans les normes de constitutionnalité applicables ou dans les circonstances, de droit ou de fait, qui affectent la portée de la disposition législative critiquée ${ }^{29}$. Une telle réserve permet de prendre en compte l'application concrète de la loi, ainsi que l'atteste la décision rendue au sujet du régime de la garde à vue ${ }^{30}$. Concernant la loi sur la dissimulation du visage dans l'espace public, un changement de circonstances paraît toutefois pour l'heure hypothétique. D'une part, un changement des circonstances de droit supposerait par exemple la reconnaissance de nouveaux droits ou principes constitutionnels afférents à l'apparence dans l'espace public. D'autre part, le recours au changement de circonstances de fait, plus délicat car emportant une certaine instabilité juridique, est destiné à n'être qu'« exceptionnel $»^{31}$. Notons toutefois que le propos du sénateur Portelli, rapporteur du projet de loi organique du 10 décembre 2009 , selon lequel «[e]n particulier, un changement de circonstances de fait ne semble admissible que plusieurs décennies après l'adoption de la disposition législative litigieuse $»^{32}$ apparaît exagéré. En matière de garde à vue, il a en

28. Décision $n^{\circ}$ 2010-9 QPC du 2 juillet 2010, Section française de l'Observatoire international des prisons, considérants 4 et 5 . Nous soulignons.

29. Décision précitée $\mathrm{n}^{\circ}$ 2009-595 DC du 3 décembre 2009 (considérant 13).

30. Décision $n^{\circ}$ 2010-14/22 QPC du 30 juillet 2010, Daniel W. et autres.

31. H. Portelli, rapport $n^{\circ} 637$ (2008-2009), fait au nom de la commission des lois du Sénat, sur le projet de loi organique introduisant la question prioritaire de constitutionnalité: "Le recours au changement de circonstances ne devrait, en conséquence, intervenir que de manière tout à fait exceptionnelle».

32. Ibid. Nous soulignons. 
effet été considéré que les évolutions constatées «depuis 1993» «justifi[aient] un réexamen de la constitutionnalité des dispositions contestées $»^{33}$. Dans le même ordre d'idées, une «banalisation» des pratiques tendant à dissimuler son visage dans l'espace public et conduisant à un nouvel examen de la loi par le Conseil constitutionnel n'est pas impossible. On peut toutefois, pour l'heure, la considérer comme aléatoire.

Partant, une question prioritaire de constitutionnalité ne serait probablement pas acceptée en vertu des articles 23-2 et 23-5 de la loi organique du 10 décembre 2009, quelle que soit l'argumentation juridique et quels que soient les moyens invoqués. Cela n'a en soi rien de fondamentalement critiquable. Le fait que, en l'absence de changement de circonstances, le Conseil constitutionnel ne soit pas conduit à «examine[r] deux fois la même chose $»^{34}$ est en effet cohérent et raisonnable. Les modalités d'articulation des contrôles a priori et a posteriori entraînent néanmoins ici une adhésion moins immédiate.

D'une part, les limites inhérentes au contrôle abstrait et objectif ne sont point corrigées, alors même que l'application effective de la loi du 11 octobre 2010 est aujourd'hui l'objet de nombreuses questions. La saisine préventive a ainsi court-circuité le débat contradictoire sur la constitutionnalité de la loi. L'appréciation concrète des arguments des requérants, potentielles parties au procès constitutionnel, n'a pas eu sa place dans le cadre d'un contrôle abstrait, détaché de tout litige. En conséquence, le respect dû aux droits de la défense, qui «découle [désormais] de l'article 16 de la Déclaration de $1789 »^{35}$,

33. Décision précitée $n^{\circ}$ 2010-14/22 QPC du 30 juillet 2010, Daniel W. et autres (considérants 13 à 18). En dépit de la décision rendue sur le régime de la garde à vue le 11 août 1993, le Conseil constitutionnel a déclaré recevable la question prioritaire de constitutionnalité portant sur le régime de droit commun de la garde à vue en raison du changement de circonstances constaté: il considère ainsi «que ces évolutions ont contribué à banaliser le recours à la garde à vue, y compris pour des infractions mineures; qu'elles ont renforcé l'importance de la phase d'enquête policière dans la constitution des éléments sur le fondement desquels une personne mise en cause est jugée; que plus de 790000 mesures de garde à vue ont été décidées en 2009; que ces modifications des circonstances de droit et de fait justifient un réexamen de la constitutionnalité des dispositions contestées».

34. Ainsi le sénateur et futur membre du Conseil constitutionnel Michel Charasse, à l'occasion de la discussion du projet de révision constitutionnelle, JO Sénat, 24 juin 2008, p. 3355, cité par B. Genevois, "Le contrôle a priori de constitutionnalité au service du contrôle a posteriori. À propos de la décision $\mathrm{n}^{\circ}$ 2009-595 DC du 3 décembre 2009 ", RFDA, 2010, 1, p. 1 s.

35. Décision $n^{\circ}$ 2010-32 QPC du 22 septembre 2010, M. Samir M. et autres [Retenue douanière], considérant 5 . Notons que, après avoir été initialement défini comme «résult[ant] des principes fondamentaux reconnus par les lois de la République » (Décision n 76-70 DC du 02 décembre 1976 - Loi relative au développement de la prévention des accidents du travail), «le respect du principe 
en souffre indirectement. À cet égard, l'autorité générale et définitive conférée à la décision du Conseil constitutionnel - sauf changement de circonstances, on l'a dit, très improbable en l'espèce - peut être critiquée.

D'autre part, le malaise provient de la combinaison d'une saisine très (trop?) large et d'une décision très (trop?) concise: en l'absence d'un dispositif tirant les conséquences précises de griefs expressément formulés, la décision de conformité soulève plus de questions qu'elle n'apporte de réponses.

Une telle parcimonie dans la motivation juridique laisse subsister une incertitude quant au fondement juridique de l'interdiction de la dissimulation du visage. En conséquence, une incertitude demeure également quant à la qualité juridique de la loi.

\section{Une décision juridiquement décevante}

Au terme d'un contrôle de proportionnalité a minima, le Conseil constitutionnel s'est contenté de constater que, à l'exception d'une réserve, la loi déférée ne porte pas d'atteinte manifeste aux libertés fondamentales. Une telle parcimonie dans la motivation juridique laisse subsister une incertitude quant à la qualité juridique de la loi.

\section{Un contrôle de proportionnalité a minima}

Selon le cinquième considérant de la décision du 7 octobre 2010, «le législateur a adopté des dispositions qui assurent, entre la sauvegarde de l'ordre public et la garantie des droits constitutionnellement protégés, une conciliation qui n'est pas manifestement disproportionnée». Ainsi que le confirme le commentaire aux Cahiers $^{36}$, le Conseil constitutionnel a déclaré conforme la loi interdisant la dissimulation du visage dans l'espace public en se plaçant sur le terrain du contrôle de proportionnalité. C'est là un terrain traditionnel de l'encadrement des ingérences étatiques dans l'exercice des libertés, bien connu du

des droits de la défense» avait ensuite été rattaché à l'article 16 de la Déclaration des droits de l'homme et du citoyen de 1789 de manière moins directe (décision n 2010-15-23 QPC du 23 juillet 2010, Région Languedoc-Roussillon et autres [Article 575 du code de procédure pénale]).

36. Les Cahiers du Conseil constitutionnel, Cahiers $n^{\circ} 30$. (http://www.conseil-constitutionnel.fr/ conseil-constitutionnel/root/bank/download/2010-613DC-ccc_613dc. pdf). 
juge administratif et de la Cour européenne des droits de l'homme. Il s'agit de s'assurer de la conciliation des principes constitutionnels en présence, droits et libertés fondamentaux objets de l'ingérence d'une part, principes invoqués pour justifier l'interdiction d'autre part. Le raisonnement suivi conduit, au terme d'un contrôle très restreint, à justifier la compétence du législateur pour encadrer l'exercice des libertés.

\section{Un contrôle de l'erreur manifeste, dans la lignée d'une tendance actuelle à la régression du contrôle de proportionnalité}

Certaines décisions récentes rendues par le Conseil constitutionnel marquent une évolution vers un affinement du contrôle de proportionnalité exercé $^{37}$. Celui-ci est ainsi désormais susceptible d'intégrer divers éléments. Le contrôle de l'adéquation des moyens au but poursuivi, combiné au contrôle de nécessité tendant à vérifier l'inexistence de mesure plus appropriée, traduit notamment l'influence des techniques utilisées par la Cour constitutionnelle allemande ${ }^{38}$. En outre, le degré du contrôle exercé, entier ou restreint à la sanction des erreurs manifestes, peut varier en fonction notamment de l'importance du droit constitutionnel en cause - sans que les critères de cette variation du contrôle soient clairement établis.

$\mathrm{Au}$ rebours de cette «subtilisation [progressive de la jurisprudence] - au sens d'un processus allant vers davantage de subtilité $-»^{39}$, le contrôle opéré

37. De manière générale, sur ces questions, se reporter aux analyses de V. Goesel-Le Bihan : « Réflexion iconoclaste sur le contrôle de proportionnalité exercé par le Conseil constitutionnel », R.F.D.C., 1997, p. 2275.; "Le contrôle exercé par le Conseil constitutionnel: défense et illustration d'une théorie générale », R.F.D.C., 2001, p. 67s.; "Le contrôle de proportionnalité dans la jurisprudence du Conseil constitutionnel: figures récentes», R.F.D.C., 2007, p. 275 s.

38. Sont généralement citées dans ce sens la décision n²008-562 DC du 21 février 2008 - Loi relative à la rétention de sûreté et à la déclaration d'irresponsabilité pénale pour trouble mental et la décision 2009-580 DC du 10 juin 2009 - Loi favorisant la diffusion et la protection de la création sur internet (dite loi Hadopi). Il est au reste tout à fait intéressant de constater que, dans le cadre de l'examen de la loi sur la rétention de sûreté, le Conseil constitutionnel s'est directement penché sur la jurisprudence de la Cour constitutionnelle allemande afférente à cette question, allant jusqu'à faire traduire les décisions des juges allemands. Se reporter à ce sujet au dossier documentaire: http://www.conseil-constitutionnel.fr/conseil-constitutionnel/root/bank/download/2008-562DC-doc. pdf (et spéc. p. 65s.).

Sur l'influence de la Cour de Karlsruhe: C. Grewe, "Les influences du droit allemand des droits fondamentaux sur le droit français: le rôle médiateur de la jurisprudence de la Cour européenne des droits de l'homme», RUDH, 2004, p. 26-32.

39. V. Goesel-Le Bihan, "Le contrôle de proportionnalité exercé par le Conseil constitutionnel: Présentation générale», $L P A, 5$ mars $2009 n^{\circ}$ 46, p. 63. 
dans la décision du 7 octobre 2010 est explicitement réduit aux seules disproportions manifestes. Il n'est, en premier lieu, pas assorti de la recherche d'une mesure alternative constitutive d'une moindre ingérence dans les libertés concernées. Le Conseil constitutionnel a, à ce titre, maintes fois rappelé qu'il «ne dispose pas d'un pouvoir général d'appréciation et de décision identique à celui du Parlement $»^{40}$. Prise à la lettre, cette formule paraît interdire tout contrôle approfondi de la nécessité de la mesure, dès lors qu'un tel contrôle suppose par définition que le juge puisse aller jusqu'à substituer son appréciation à celle de l'auteur de l'acte, en vérifiant qu'une mesure moins restrictive de la liberté soit à même de permettre d'atteindre, avec la même efficacité, le même objectif constitutionnel. En tout état de cause, le contrôle dit de proportionnalité effectué est entendu de manière plus restrictive que dans la jurisprudence constitutionnelle allemande: il n'est nullement vérifié qu'il existe un rapport raisonnable entre la gravité des effets produits par l'ingérence et $l^{\prime}$ objectif poursuivi ${ }^{41}$.

Le choix ostensible d'un contrôle restreint doit, en deuxième lieu, être souligné. Cette décision se situe à cet égard dans la lignée d'une évolution constatée lorsque sont en cause des atteintes à la liberté individuelle. S'il peut «paraîtr[e] rationnel d'avancer que le contrôle entier - tout comme pour le juge administratif - est le contrôle normal $»^{42}$, il semble qu'il faille aujourd'hui «compter avec de possibles régressions lorsque la protection des droits se heurte à des impératifs jugés prioritaires $»^{43}$. Une pareille évolution s'observe en présence d'atteintes légères à la liberté individuelle stricto sensu, telle que protégée par l'article 66 de la Constitution. Pour les composantes de la liberté individuelle lato sensu, qu'il s'agisse de la vie privée ou de la liberté d'aller et de venir, protégées au titre de l'article 2 de la Déclaration, il semble d'ailleurs que le Conseil constitutionnel s'en tienne désormais à une censure des erreurs manifestes, sans distinguer selon la gravité de l'atteinte. On peut toutefois

40. Décision n 74-54 DC du 15 janvier 1975 - Loi relative à l'interruption volontaire de la grossesse (considérant 1). Jurisprudence constante.

41. Tel que mis en œuvre par la Cour constitutionnelle allemande, le principe de proportionnalité suppose de vérifier le caractère approprié de la mesure - aptitude à atteindre les objectifs poursuivis -, sa nécessité - inexistence d'autres moyens affectant de manière moins préjudiciable les personnes et la collectivité -, et enfin sa proportionnalité au sens étroit, selon laquelle la mesure ne saurait être hors de proportion avec le résultat recherché. Voir par exemple: M. Fromont, "Le principe de proportionnalité », AJDA, 1995, p. $156 \mathrm{~s}$.

42. P. Wachsmann, Libertés publiques, Paris, Dalloz, 6e éd., 2009, p. 119.

43. V. Goesel-Le Bihan, "Le contrôle de proportionnalité exercé par le Conseil constitutionnel: Présentation générale, op. cit., p. $62 \mathrm{~s}$. 
s'interroger sur un contrôle de proportionnalité qui varierait en fonction de l'ingérence, alors même que celle-ci constitue l'un des plateaux de la balance.

«Signe du temps ${ }^{44}, l^{\prime}$ inflexion dans le sens d'une telle restriction du contrôle de proportionnalité pratiqué en matière d'atteintes à la liberté individuelle et, au-delà, d'atteintes à la liberté personnelle déduite des articles 2 et 4 de la Déclaration de 1789, est observée depuis le début des années 2000. Dans sa décision relative à la loi sur la sécurité intérieure du 13 mars 2003, le Conseil relève ainsi que la «conciliation» opérée par le législateur «entre le respect de la vie privée et la sauvegarde de l'ordre public» «n'est pas manifestement déséquilibrée » ${ }^{45}$. De même, il a simplement vérifié que l'interdiction générale de porter une tenue destinée à dissimuler son visage dans l'espace public n'est pas manifestement hors de proportion avec le résultat visé.

Relevons en troisième lieu le mouvement curieux qui conduit à mettre en balance la "garantie des droits constitutionnellement protégés» avec la «nature de la peine instituée en cas de méconnaissance de la règle fixée par [le législateur] ». Sans doute le Conseil constitutionnel a-t-il apprécié l'absence de disproportion de l'ingérence au regard de la légèreté de la peine encourue en cas de violation de l'interdiction posée par la loi, soit une «amende prévue pour les contraventions de la deuxième classe» (article 3 de la loi, rappelé dans le deuxième considérant). Pour autant, la motivation extrêmement sommaire de la décision ne permet pas de lever l'incertitude tenant à l'objet de cette mise en balance, principe même de l'infraction ou détermination de la sanction qui en assortit la méconnaissance.

44. Voir notamment les analyses de V. Goesel le Bihan, qui souligne l'évolution observée «depuis le 11 septembre 2001 et afin de répondre à la menace terroriste grandissante». Ibid, p. $72 \mathrm{~s}$.

45. Décision $n^{\circ}$ 2003-467 DC du 13 mars 2003 (considérant 27). Pour la liberté d'aller et de venir, voir aussi les considérants 70 et 71. Voir, dans le même sens, les décisions n 2005-532 DC du 19 janvier 2006 - Loi relative à la lutte contre le terrorisme et portant dispositions diverses relatives à la sécurité et aux contrôles frontaliers (considérant 21); $\mathrm{n}^{\circ}$ 2007-557 DC du 15 novembre 2007 - Loi relative à la maîtrise de l'immigration, à l'intégration et à l'asile (considérant 11). La "conciliation des exigences de l'ordre public et de la garantie de libertés constitutionnellement protégées » semble au contraire avoir un temps fait l'objet d'un contrôle entier. Voir dans ce sens la décision n 94-352 DC du 18 janvier 1995 - Loi d'orientation et de programmation relative à la sécurité.

On notera qu'aucune des décisions mentionnées dans le dossier documentaire accompagnant la décision du 7 octobre 2010 ne s'écarte de cette tendance: Décision nº 99-411 DC du 16 juin 1999 - Loi portant diverses mesures relatives à la sécurité routière et aux infractions sur les agents des exploitants de réseau de transport public de voyageurs; Décision n² 2003-467 DC du 13 mars 2003Loi pour la sécurité intérieure; Décision $\mathrm{n}^{\circ} 2009-580$ DC du 10 juin 2009 - Loi favorisant la diffusion et la protection de la création sur internet; Décision n 2010-605 DC du 12 mai 2010 - Loi relative à l'ouverture à la concurrence et à la régulation du secteur des jeux d'argent et de hasard en ligne. 
Sauf à établir l'existence d'une disproportion manifeste, le Conseil constitutionnel est amené à entériner les choix du législateur.

\section{Le poids incertain d'un ordre public «social» avancé par le législateur dans la balance constitutionnelle}

Si la balance des intérêts à laquelle il est procédé est somme toute classique, l'analyse des deux "plateaux de la balance» constitutionnelle appelle certaines observations.

Nonobstant la réserve d'interprétation afférente aux lieux de culte ouverts au public - fondée sur la liberté religieuse -, le Conseil constitutionnel ne précise nullement les «droits constitutionnellement protégés» potentiellement atteints (considérant 5). Ni la liberté d'aller et venir, ni le respect de la vie privée déduit de l'article 2 de la Déclaration de 1789, ni encore la liberté d'expression, telle que garantie par son article 11, pourtant mentionnés dans le commentaire aux Cahiers, ne sont évoqués. Les dispositions sur lesquelles le contrôle est fondé soulignent en revanche la compétence du législateur pour encadrer les libertés (considérant 3). L'article 4 de la Déclaration de 1789 dispose ainsi que les «bornes » posées à «l'exercice des droits naturels de chaque homme» «ne peuvent être déterminées que par la loi», laquelle «n'a le droit de défendre que les actions nuisibles à la société» (article 5), afin que «l'ordre public établi par la loi» (article 10) soit respecté. En choisissant de se référer à ces dernières normes, le Conseil rappelle que le constituant lui-même a entendu laisser au législateur une marge d'appréciation, y compris en assignant des limites aux libertés proclamées. Or, dans cet arbitrage entre libertés constitutionnelles, objectifs de valeur constitutionnelle fondant des limites législatives à ces libertés et limites de ces limitations législatives, le rôle de la justice constitutionnelle est aussi délicat qu'il est décisif.

L'objectif de protection de l'ordre public, objectif de valeur constitutionnelle ${ }^{46}$, est précisément l'élément fondamental concourant à encadrer les libertés constitutionnelles - sans que le Conseil constitutionnel n'opère de hiérarchie entre ces différentes composantes du bloc de constitutionnalité.

$C^{\prime}$ est donc sans surprise que «le législateur» a invoqué «des fins de protection de l'ordre public» (considérant 4) pour justifier l'interdiction de la dissimulation du visage dans l'espace public. Il est établi que le recours au principe de laïcité, compris comme la neutralité des pouvoirs publics envers les religions, peut difficilement justifier une interdiction étendue à tout «l'es-

46. Pour la «constitutionnalisation» de l'ordre public: Décision n 80-127 DC du 20 janvier 1981 - Loi renforçant la sécurité et protégeant la liberté des personnes (considérant 56); Décision no 82-141 DC du 27 juillet 1982 - Loi sur la communication audiovisuelle (considérant 5). 
pace public $»^{47}$, qui plus est au-delà de toute référence à une expression religieuse. De même, le respect de la dignité humaine ne semble pas davantage en mesure d'autoriser l'interdiction de la dissimulation du visage ${ }^{48}$. La portée de ce principe constitutionnel, posé par le Conseil constitutionnel dans ses décisions sur les lois «bioéthique» et déduit des intentions manifestées par les auteurs du Préambule de la Constitution du 27 octobre $1946^{49}$ demeure en effet controversée. Dans sa célèbre décision Commune de Morsang-sur-Orge $e^{50}$, le Conseil d'État a certes admis que la transgression de ce qu'il a érigé en composante «immatérielle» de l'ordre public puisse justifier une mesure d'interdiction, rejetant comme inopérante l'invocation de la liberté individuelle. Consulté par le gouvernement sur les "possibilités juridiques d'interdiction du port du voile intégral», le même Conseil d'État a rappelé la fragilité de ce concept, «en particulier dans le cas où le port du voile intégral résulte de la volonté délibérée d'une personne majeure ${ }^{51}$. Une acception mettant l'accent sur le droit au respect de l'autonomie de la volonté et de la vie privée, rejetant ce faisant une définition objective, dont les contours seraient dessinés par l'État, est au demeurant davantage conforme à la jurisprudence de la Cour de Strasbourg 52 .

C'est à une dimension plus large de l'ordre public que renvoie la justification invoquée par le législateur. Dans l'exposé des motifs de la loi, il est précisé que «la défense de l'ordre public ne se limite pas à la préservation

47. La Cour européenne des droits de l'homme l'a affirmé de manière très nette dans l'arrêt Arslan c/ Turquie devenu définitif (CEDH, 23 févr. 2010, $\mathrm{n}^{\circ}$ 41135/98).

48. Dans un sens contraire: B. Mathieu, "La validation par le Conseil constitutionnel de la loi sur "le voile intégral". - La reconnaissance implicite d'un ordre public "immatériel” ", JCP G, $\mathrm{n}^{\circ} 42$, 18 octobre 2010, p. 1018.

49. Décision précitée $n^{\circ}$ 94-343/344 DC du 27 juillet 1994. II «ressort [du Préambule de la Constitution de 1946] que la sauvegarde de la dignité de la personne humaine contre toute forme d'asservissement et de dégradation est un principe à valeur constitutionnelle ».

50. CE, Ass., 27 octobre 1995, Commune de Morsang-sur-Orge, $n^{\circ}$ 136727, Lebon 372; AJDA, 1995, p. 942, chronique J.-H. Stahl et D. Chauvaux; RFDA, 1995, p. 1204, concl. P. Frydman. Notons que cette décision est citée dans le dossier documentaire accompagnant la décision du 7 octobre 2010.

51. CE, Section du rapport et des études, Étude relative aux possibilités juridiques d'interdiction du port du voile intégral, rapport adopté par l'assemblée générale plénière, le 25 mars 2010 (http:// www.conseil-etat.fr/cde/fr/rapports-et-etudes/possibilites-juridiques-d-interdiction-du-port-du-voileintegral.html).

Voir à ce sujet: A. Levade, «Le Conseil d'État aux prises avec le voile intégral - Les enjeux d'un refus du risque juridique », JCP G, $\mathrm{n}^{\circ} 15,12$ avril 2010, p. 406.

52. CEDH, 29 avril 2002, $\mathrm{n}^{\circ}$ 2346/02, Pretty c/ Royaume-Uni (Rec. CEDH, 2002, III, § 66); CEDH, 17 février 2005, n 42758/98, K. A. et A. D. c/ Belgique. 
de la tranquillité, de la salubrité ou de la sécurité. Elle permet également de prohiber des comportements qui iraient directement à l'encontre de règles essentielles au contrat social républicain, qui fonde notre société $»^{53}$. L'appel à la prudence formulé par le Conseil d'État à l'endroit d'une conception « renouvelée et élargie» de l'ordre public ${ }^{54} \mathrm{n}^{\prime}$ a donc pas été entendu.

Pour autant, le raisonnement suivi par le Conseil constitutionnel pour entériner cette justification demeure peu explicite. Les contours de l'ordre public ne sont pas expressément tracés. Dès lors que la dissimulation du visage entrave l'identification, son interdiction peut répondre à un impératif d'ordre public, classiquement défini comme matériel et extérieur ${ }^{55}$. Le quatrième considérant fait ainsi référence à un «danger pour la sécurité publique». À l'égard d'une prohibition générale s'étendant à tout «l'espace public», cette interprétation est toutefois sujette à caution. Faut-il alors y voir la consécration d'un ordre public «immatériel $»^{56}$, «social», tendant à sauvegarder «les exigences minimales de la vie en société», également mentionnées dans le quatrième considérant? La dimension «morale» de l'ordre public, certes présente dans la jurisprudence du Conseil d'État, mais rapportée à des circonstances concrètes de temps et de lieu ${ }^{57}$, trouverait ainsi l'une de ses expressions dans la défense de l'atteinte «immorale» aux valeurs de la République constituée par l'oc-

53. Loi $n^{\circ}$ 2010-1192 du 11 octobre 2010 interdisant la dissimulation du visage dans l'espace public. http://www.legifrance.gouv.fr/affichLoiPubliee.do; jsessionid=53621F74407CE51750F6oD8BoC2B49D 1.tpdj014v_2 ?idDocument=JORFDOLEooo022234691\&type=expose. Voir aussi sur ce point le rapport sur le projet de loi, présenté par J.-P. Garraud, n 2520 , http://www.assemblee-nationale.fr/13/ rapports/r2648.asp\#P165_29087.

54. Conseil d'État, rapport précité, Étude relative aux possibilités juridiques d'interdiction du port du voile intégral, p. 26. Voir sur ce point, D. de Béchillon, «Voile intégral: éloge du Conseil d’État en théoricien des droits fondamentaux», RFDA, 2010, p. 467s.

55. CE, 27 juillet 2001, Fonds de défense des musulmans en justice, $\mathrm{n}^{\circ}$ 216903, Lebon, p. 400: la subordination de la délivrance des documents d'identité à la présentation d'une photographie, «face et tête nue» apporte sans doute une restriction à l'expression de la liberté religieuse, s'agissant du port du voile pour une musulmane. Mais une telle restriction n'est pas disproportionnée au regard de l'objectif de limiter les risques de falsification et d'usurpation d'identité, et au regard de la délimitation de son objet. Voir aussi les analyses de R. Hanicotte, "La dissimulation du visage au regard de l'ordre public», $A J D A, 2010$, p. 417.

56. B. Mathieu, "La validation par le Conseil constitutionnel de la loi sur "le voile intégral". - La reconnaissance implicite d'un ordre public “immatériel”, op. cit., p. 1018; Voir aussi sur ce point: F. Dieu, "Le droit de dévisager et l'obligation d'être dévisagé: vers une moralisation de l'espace public?», JCP A, n 48, 29 novembre 2010, p. 2355.

57. CE, 18 décembre 1959, Société "Les films Lutetia», n 36385, Lebon 693. Cette décision est au reste également mentionnée dans le dossier documentaire accompagnant la décision du 7 octobre 2010. Voir aussi CE, Ord., 8 juin 2005, Commune de Houille, ${ }^{\circ} 281084$. 
cultation du visage, dans une République «qui se vit à visage découvert». Le recours à l'article 5 de la Déclaration du 26 août 1789 , selon lequel «la loi n'a le droit de défendre que les actions nuisibles à la Société», pour justifier l'interdiction posée par la loi, prend ici tout son sens ${ }^{58}$. La réglementation du "vivre ensemble» par le législateur est d'autant plus facilement entérinée que les libertés qui pourraient en constituer les bornes ne sont pas mentionnées dans la décision. Le Conseil constitutionnel est ainsi porté à prendre acte de la volonté de ce dernier, constitutionnellement habilité à protéger la société contre des comportements individuels qui lui seraient nuisibles.

L'exercice de conciliation présenté s'apparente principalement à un exposé de ce que «le législateur a estimé» bon de faire. Dans un long quatrième considérant, il est renvoyé à «l'objet» des dispositions de la loi, laquelle «tend à répondre à l'apparition de pratiques, jusqu'alors exceptionnelles, consistant à dissimuler son visage dans l'espace public». L'appréciation du législateur qui «a estimé que de telles pratiques peuvent constituer un danger pour la sécurité publique et méconnaissent les exigences minimales de la vie en société [...et] que les femmes dissimulant leur visage, volontairement ou non, se trouvent placées dans une situation d'exclusion et d'infériorité manifestement incompatible avec les principes constitutionnels de liberté et d'égalité» est simplement relevée. On notera à cet égard la faible distance établie par le Conseil constitutionnel entre les normes constitutionnelles applicables d'un côté, l'analyse des dispositions de la loi de l'autre ${ }^{59}$. Cet exposé des «objectifs que [le législateur] s'est assignés » (considérant 5) conduit presque naturellement le Conseil à ratifier la "généralis[ation] des règles jusque là réservées à des situations ponctuelles à des fins de protection de l'ordre public» (considérant 4), généralisation pourtant précisément objet de toutes les réserves juridiques.

On remarquera de surcroît une certaine confusion entre l'élargissement de l'objet de la loi à toute tenue destinée à dissimuler le visage et la référence indirecte au port de la burqa ou du niqab "par les femmes». L'exposé, par le Conseil constitutionnel, des justifications invoquées par le législateur conduit

58. Bertrand Mathieu souligne à cet égard que «cette référence est d'autant plus remarquable que le Conseil n'en a fait application qu'à une seule occasion et de manière que l'on pourrait qualifier de fortuite» (Décision $n^{\circ}$ 2000-426 DC du 30 mars 2000 - Loi relative à la limitation du cumul des mandats électoraux et des fonctions et à leurs conditions d'exercice (considérant 5)). B. Mathieu, "La validation par le Conseil constitutionnel de la loi sur "le voile intégral". - La reconnaissance implicite d'un ordre public “immatériel” », op. cit., p. 1018.

59. Voir dans ce sens: M. Verpeaux, «Dissimulation du visage, la délicate conciliation entre la liberté et un nouvel ordre public», AJDA, 2010 p. 2373. 
à mettre en lumière la raison «dissimulée» derrière la généralité des termes de la loi.

La lecture de la décision du 7 octobre 2010 ne permet pas réellement de dissiper ces confusions. Si la motivation retenue pour valider l'interdiction générale était particulièrement attendue, la disproportion entre la volonté de désamorcer toute polémique juridique et la faiblesse de la motivation de la décision établissant la constitutionnalité de la loi est patente.

\section{La persistance d'une incertitude juridique}

La persistance d'une incertitude juridique tient tant à l'hermétisme de la décision du Conseil constitutionnel qu'aux doutes quant à la conventionnalité de la loi.

\section{Un hermétisme jurisprudentiel contestable}

La distance entre l'avis défavorable rendu par le Conseil d'État sur le projet de loi et la décision du Conseil constitutionnel déclarant la loi conforme a été maintes fois relevée. Saisi par le gouvernement, le premier a rendu un avis nuancé, guidé par son hostilité de principe à l'encontre des mesures générales et absolues ${ }^{60}$. Partant, il s'est efforcé de préconiser des interdictions partielles, fondées sur les exigences de la sécurité publique, ou encore sur des impératifs de lutte contre la fraude, justifiant l'obligation de découvrir son visage ${ }^{61}$. La seule référence à cet avis, présentée dans le commentaire aux Cahiers, concerne la réserve d'interprétation que le Conseil d'État formule lui-même à l'endroit des lieux de culte.

Ce qui frappe finalement, c'est le caractère laconique de la motivation de la décision. Il est déconcertant de constater que le renvoi au «commentaire aux Cahiers» accompagnant la publication électronique de la décision se voit attribuer en quelque sorte le rôle d'éclaircir le raisonnement suivi. On regrette ainsi l'absence d'un contrôle qui établirait expressément dans quelle mesure l'atteinte générale à la liberté personnelle issue des articles 2 et 4 de la Déclaration des droits de l'homme et du citoyen et à celle de manifester ses opinions, garantie par son article 10, est proportionnée au risque de trouble à l'ordre public, compte tenu des dispositifs existants.

6o. On cite ainsi classiquement les conclusions du commissaire du gouvernement Corneille sur CE, Sect., 10 août 1917, Baldy: «la liberté est la règle et la restriction de police, l'exception ».

61. Rapport précité, spéc. p. 37. 
Il est en effet possible de tenir le raisonnement selon lequel, une fois découplée de toute motivation religieuse, la «dissimulation » intégrale du visage n'a pas sa place dans un espace "public». Révélant une «fermeture» à l'espace public, par définition «ouvert», elle entre en contradiction avec la nécessité qui y prévaut de pouvoir identifier toute personne. On peut ainsi penser qu'il est contraire aux principes d'une société «ouverte» $d^{\prime}$ «occulter» son visage à autrui lorsque l'on se trouve sur la place "publique». L'élargissement de la notion de l'ordre public conduirait ce faisant à définir l'espace public comme un espace social imposant l'obligation minimale de pouvoir être "dévisagé», et s'imposant de la sorte au libre arbitre des individus ${ }^{62}$. À ce titre, l'absence de référence à toute circonstance spécifique de lieu ou de temps semble justifiée. De même, dans une telle approche, on comprend que le caractère général de l'interdiction trouve ses seules bornes au seuil de l'espace "privé» et des «lieux de culte ouverts au public», objet de la réserve d'interprétation opposée à la loi (considérant 5). Ce serait là tout l'intérêt de la définition législative d'un espace "constitué des voies publiques ainsi que des lieux ouverts au public [commerces, transports, etc.] ou affectés à un service public [mairies, écoles, etc.]».

La définition est avalisée par le Conseil constitutionnel. Reste que, après la virulence des débats politiques ayant précédé l'adoption de la loi, la quête de légitimité juridique des décisions du Conseil constitutionnel aurait supposé qu'il étayât davantage sa consécration des choix du législateur. Il est régulièrement rappelé que le caractère juridique de l'argumentation développée par le Conseil constitutionnel constitue l'élément essentiel du renforcement de sa légitimité. Dans une démocratie représentative, la motivation des jugements constitue un élément essentiel de la discussion des décisions, «d'autant plus centra[1] que la juridiction constitutionnelle s'empare de la loi, dont l'élaboration, dans un tel système, présuppose publicité et discussion ${ }^{63}$.

Quelle que soit l'appréciation que l'on peut porter in fine sur l'action du législateur, on peut déplorer le caractère lapidaire d'une motivation qui ne parvient pas à dissiper les doutes juridiques. Il restera intéressant d'observer le sort que les juridictions ordinaires, puis, le cas échéant, la Cour européenne des droits de l’homme réserveront à la loi.

62. Dans ce sens: F. Dieu, «Le droit de dévisager et l'obligation d'être dévisagé: vers une moralisation de l'espace public?», op. cit., p. 2355.

63. O. Jouanjan, «Modèles et représentations de la justice constitutionnelle en France: un bilan critique», Jus Politicum, 2, 2009, http://www.juspoliticum.com/Modeles-et-representations-de-la.html. 


\section{La conventionnalité de la loi en suspens}

L'établissement de la conformité à la Constitution laisse libre cours aux questions relatives à sa conventionnalité.

La portée de la saisine préventive du Conseil constitutionnel concernant une éventuelle question prioritaire de constitutionnalité a déjà été soulignée. On sait que, lorsqu'une juridiction nationale est saisie de moyens contestant la conformité d'une disposition législative aux droits et libertés garantis par la Constitution d'une part, aux engagements internationaux de la France d'autre part, elle doit se prononcer par priorité sur la question de constitutionnalité64. Rien ne s'oppose néanmoins à ce qu'un juge national confronte la loi à la Convention européenne de sauvegarde des droits de $1^{\prime}$ homme et des libertés fondamentales ${ }^{65}$. Ainsi que le Conseil constitutionnel l'a lui-même spécifié, l'autorité qui s'attache à ses décisions «ne limite pas la compétence des juridictions administratives et judiciaires pour faire prévaloir [1]es engagements [internationaux ou européens de la France] sur une disposition législative incompatible avec eux, même lorsque cette dernière a été déclarée conforme à la Constitution ${ }^{66}$. Le caractère "prioritaire» de l'examen de la question de constitutionnalité n'exclut en rien le prononcé ultérieur d'une inconventionnalité par le juge "ordinaire», en charge de l'appréciation concrète de la conformité de la loi au regard des dispositions conventionnelles.

Par voie de conséquence, il est légitime de s'interroger sur la conventionnalité de la loi, que cette question soit soulevée devant les juges français ou, le cas échéant, devant la Cour de Strasbourg, une requête devant cette dernière étant, à moyen terme, probable.

64. Article 23-2 de la loi organique $\mathrm{n}^{\circ}$ 2009-1523 du 10 décembre 2009 relative à l'application de l'article 61-1 de la Constitution (précitée).

65. C'est ainsi que la Cour de cassation a été amenée à juger le régime français de garde à vue contraire aux règles du procès équitable qui résultent de l'article 6 de la Convention européenne des droits de l'homme. Sa décision du 19 octobre 2010 (Cass., crim. n $^{\circ} 5699$ du 19 octobre 2010) fait suite à l'arrêt Brusco c. France rendu dans le même sens par la Cour de Strasbourg le 14 octobre 2010 (CEDH, 5 e Sect., 14 octobre 2010, Req. $n^{\circ}$ 1466/07) - question, on l'a vu, par ailleurs déjà examinée par le Conseil constitutionnel.

66. Décision du 12 mai $2010 \mathrm{n}^{\circ}$ 2010-605 DC - Loi relative à l'ouverture à la concurrence et à la régularisation du secteur des jeux d'argent et de hasard en ligne (considérant 13). Voir à ce sujet: Dalloz actualité, 19 mai 2010, obs. S. Lavric; B. Mathieu, "La guerre des juges n’aura pas lieu À propos de la décision du 12 mai 2010 du Conseil constitutionnel», JCP A, n 22, 31 mai 2010, p. 2181S. Voir aussi, dans le même sens: CE, 14 mai 2010, n 312305, Dalloz actualité, 25 mai 2010, obs. C. de Gaudemont. 
La réserve d'interprétation formulée par le Conseil constitutionnel au sujet des «lieux de culte ouverts au public » met en exergue la question des croyances religieuses à l'origine de la loi. Elle s'interprète à la lumière des exigences de l'article 9 de la Convention européenne de sauvegarde des droits de l'homme et des libertés fondamentales, tel qu'il est interprété par la jurisprudence de la Cour de Strasbourg67. La compatibilité de la loi du 11 octobre 2010 avec la Convention n'en est pas pour autant assurée. Dans son arrêt récent Arslan c/ Turquie, la Cour européenne indique en particulier que la marge d'appréciation nationale conférée aux États est réduite lorsque la manifestation publique d'une religion se déroule «dans des lieux publics ouverts à tous comme les voies ou places publiques», et non "dans des établissements publics, dans lesquels le respect de la neutralité à l'égard de croyances peut primer sur le libre exercice du droit de manifester sa religion ${ }^{68}$. On peut remarquer l'hiatus existant entre cette marge d'appréciation réduite que la Cour reconnaît aux États et la liberté d'appréciation du législateur qu'a manifestement admise le Conseil constitutionnel dans la décision commentée. Mais les enseignements de cet arrêt également éclairent le débat relatif à la conventionnalité de la loi sur le fond, dès lors que la Cour y condamne une interdiction générale emportant une ingérence étatique dans l'exercice de la liberté religieuse ${ }^{69}$. Au vu des faits spécifiques de l'espèce, ils ne le tranchent cependant pas définitivement. La tenue vestimentaire en cause était composée non pas d'attributs

67. Dans ce sens, A. Levade, «Épilogue d'un débat juridique: l'interdiction de la dissimulation du visage dans l'espace public validée!», op. cit., p. 1043. L'attention portée à la jurisprudence de la Cour de Strasbourg justifie en outre que la loi n'ait pas été prise sur le fondement du principe de laïcité. Dans ce sens: M. Verpeaux, «Dissimulation du visage, la délicate conciliation entre la liberté et un nouvel ordre public», op. cit., p. 2373.

Pour la jurisprudence de la Cour européenne des droits de l'homme, se reporter à l'exposé de J.-P. Marguénaud, "La liberté de porter des vêtements religieux dans les lieux publics ouverts à tous", Dalloz, 2010, p. 682.

68. CEDH, 23 février 2010, Ahmet Arslan e.a. c/ Turquie [Aff. 41135] (AJDA, 2010, p 362): «ll s'ensuit que la jurisprudence de la Cour mettant l'accent sur l'importance particulière du rôle du décideur national [...] ne trouve pas à s'appliquer» (§ 49). L'importance accordée au rôle du décideur national lorsque sont en jeu des questions sur les rapports entre l'État et les religions avait, à l'inverse, précisément été soulignée dans l'arrêt de grande chambre Leyla Sahin c/ Turquie du 10 novembre 2005 (AIDA, 2006, p. 315, obs. G. Gonzalez). Mais il s'agissait alors d'une réglementation du port de symboles religieux circonscrite aux établissements d'enseignement.

Voir aussi l'avis de la Commission nationale consultative des droits de l'homme: Assemblée plénière, 21 janvier 2010, Avis sur le port du voile intégral, Actualités droits-libertés du 11 février 2010.

69. Étaient en cause une loi de 1925 relative au port du chapeau et une loi de 1934 réglementant le port de certains vêtements religieux. 
dissimulant le visage mais "d'un turban, d'un "salvar" (saroual) et d'une tunique», tenue caractéristique d'une confrérie religieuse. Les requérants, membres de ce groupe, s'apprêtaient à participer à une cérémonie à caractère religieux. De plus, amenée à se prononcer sur la loi française, la Cour de Strasbourg accorderait probablement une attention spécifique à l'invocation de l'égalité des sexes, qui sous-tend l'interdiction posée, ou encore à la prise en compte des discussions relatives à la nécessité de découvrir son visage face à autrui dans l'espace public. Placée sur le terrain de la laïcité, du pluralisme religieux face à la neutralité de l'État, l'arrêt Arslan n'embrasse pas cette dernière dimension d'un ordre public «social», destiné à sauvegarder une certaine conception de la société.

Saisie d'une potentielle requête contestant une loi d'interdiction générale s'apparentant, même à demi-mots, à une ingérence du législateur dans la libre manifestation des opinions religieuses, la Cour serait amenée à vérifier que la mesure répond au critère de «nécessité dans une société démocratique». La question des contours de la notion d'ordre public invoquée par le législateur français, et entérinée par le Conseil constitutionnel, serait alors à nouveau posée.

\section{Conclusion}

Depuis l'entrée en vigueur de l'article 61-1 de la Constitution, l'intervention du Conseil constitutionnel est appelée à revêtir une plus grande importance. La décision rendue le 7 octobre 2010 rappelle cependant la retenue qui caractérise l'action du Conseil dans le champ des libertés publiques ${ }^{70}$.

$\mathrm{Au}$ terme d'un contrôle restreint somme toute classique, la loi sur l'interdiction de dissimuler son visage a été jugée conforme à la Constitution, à une réserve près. Certaines interrogations demeurent. Lorsqu'est en jeu une interdiction générale et absolue, l'équilibre des plateaux de la balance, entre la sauvegarde d'un ordre public aux dimensions plurielles et les droits constitutionnellement protégés, peut-il être assuré? La décision du Conseil constitutionnel souligne la difficulté de l'exercice d'une telle conciliation. Si le recours

70. Dans ce sens: V. Champeil-Desplats, «Le Conseil constitutionnel, protecteur des libertés ou cerbère de la production législative?», Frontières du droit, critique des droits. Billets d'humeur en l'honneur de Danièle Lochak, Paris, LGDJ, 2007, p. 2515.; P. Wachsmann, «Des chameaux et des moustiques. Réflexions critiques sur le Conseil constitutionnel», ibid., p. 2795. 
à l'article 5 de la Déclaration des droits de l'homme et du citoyen le conduit à consacrer la compétence du législateur pour encadrer les "actions nuisibles à la Société», on aurait toutefois pu attendre qu'il explicite davantage sa démarche et ses motivations. L'importance accordée au respect de la prise en compte des choix du législateur n'est pas en soi à dénoncer. Reste que, «resserré dans ses limites, le pouvoir accordé aux tribunaux [...] de se prononcer sur l'inconstitutionnalité des lois forme encore une des plus puissantes barrières qu'on ait jamais relevées contre la tyrannie des assemblées politiques $»^{71}$. Si les cours constitutionnelles sont placées au cœur du système politique et constitutionnel, c'est bien en revendiquant leur qualité de juridiction qu'elles acquièrent leur légitimité. Eu égard au renforcement des exigences démocratiques, on ne peut que relever la nécessité constante de distinguer l'argumentaire juridique de la logique politique. Les cours constitutionnelles sont d'autant plus respectées et légitimes que leurs décisions se détachent clairement des motivations prêtées au législateur et reposent elles-mêmes sur des motivations juridiques sérieuses et approfondies.

71. A. de Tocqueville, De la démocratie en Amérique (1835), II partie, chap. VII («De l’omnipotence de la majorité aux États-Unis et de ses effets»), Gallimard, «Folio/histoire», 2000, p. 369. 